\title{
Erratum to: Contribution of an additive locus to genetic variance when inheritance is multi-factorial with implications on interpretation of GWAS
}

\author{
Daniel Gianola $\cdot$ Frederic Hospital • \\ Etienne Verrier
}

Published online: 5 May 2013

(c) Springer-Verlag Berlin Heidelberg 2013

\section{Erratum to: Theor Appl Genet \\ DOI 10.1007/s00122-013-2064-2}

The authors are grateful to Yaodong Hu, Department of Animal Sciences, University of Wisconsin, Madison, for catching a missing " 2 " that propagated to several formulae, e.g., (4) and (6), and to Fig. 3, producing erroneous expressions. We apologize for the mistakes, missed throughout the review process. The paper should be modified as follows.

The online version of the original article can be found under doi:10.1007/s00122-013-2064-2.

\section{Gianola $(\bowtie)$}

Department of Animal Sciences,

University of Wisconsin-Madison,

Madison, WI 53706, USA

e-mail: gianola@ansci.wisc.edu

\section{Gianola}

Department of Animal and Aquacultural Sciences,

Norwegian University of Life Sciences, N-1432 Ås, Norway

\section{F. Hospital}

INRA, UMR1313 Génétique animale et biologie intégrative, 78350 Jouy-en-Josas, France

\section{E. Verrier}

AgroParisTech, UMR1313 Génétique animale et biologie intégrative, 75231 Paris 05, 16 Rue Claude Bernard, France
1. Formula (4) should be replaced by:

$$
\begin{aligned}
\operatorname{Var}\left(u_{i}\right)= & 2 \sum_{k=1}^{K} p_{k}\left(1-p_{k}\right) a_{k}^{2}+2 \sum_{k=1}^{K} \sum_{l=k+1}^{K} \operatorname{Cov}\left(W_{i k}, W_{i l}\right) a_{k} a_{l} \\
= & 2 \sum_{k=1}^{K} p_{k}\left(1-p_{k}\right) a_{k}^{2} \\
& +2 \sum_{k=1}^{K} \sum_{l=k+1}^{K} 2 \rho_{k l} \sqrt{p_{k}\left(1-p_{k}\right) p_{l}\left(1-p_{l}\right)} a_{k} a_{l} \\
= & 2 \sum_{k=1}^{K} p_{k}\left(1-p_{k}\right) a_{k}^{2}+2 \sum_{k=1}^{K} \sum_{l=k+1}^{K} 2 D_{k l} a_{k} a_{l} .
\end{aligned}
$$

2. Four lines below formula (4), replace

$$
\begin{aligned}
& \rho_{k l}=\frac{2 D_{k l}}{\sqrt{p_{k}\left(1-p_{k}\right) p_{l}\left(1-p_{k l}\right)}}, \\
& \text { by } \\
& \rho_{k l}=\frac{D_{k l}}{\sqrt{p_{k}\left(1-p_{k}\right) p_{l}\left(1-p_{k l}\right)}} .
\end{aligned}
$$

3. Replace formula (6) by

$$
\begin{aligned}
C_{j}= & 2 \rho_{j 1} \sqrt{p_{j}\left(1-p_{j}\right) p_{1}\left(1-p_{1}\right)} a_{j} a_{1} \\
& +2 \rho_{j 2} \sqrt{p_{j}\left(1-p_{j}\right) p_{2}\left(1-p_{2}\right)} a_{j} a_{2}+\cdots \\
& +2 p_{j}\left(1-p_{j}\right) a_{j}^{2}+\cdots+2 \rho_{j K} \sqrt{p_{j}\left(1-p_{j}\right) p_{K}\left(1-p_{K}\right)} a_{j} a_{K} .
\end{aligned}
$$

4. In the paragraph following equation (11), replace the passage: "As a simple illustration....makes an overstatement if disequilibrium is negative." by:

"As a simple illustration consider a 3-locus model with same allelic frequency $p$ and additive effect $a$ at each locus. Then expression (4) is

$$
\begin{aligned}
\operatorname{Var}\left(u_{i}\right) & =2 p(1-p) a^{2}\left(3+2 \rho_{12}+2 \rho_{13}+2 \rho_{23}\right) \\
& =6 p(1-p) a^{2}(1+2 \rho),
\end{aligned}
$$


where $\rho$ is the average of the three possible correlations. Here, $\operatorname{Var}_{E Q}(u)=6 p(1-p) a^{2}$ and $D_{\text {diseq }}=12 p(1-p) a^{2} \rho$. Further

$$
\begin{aligned}
V_{1} & =2 p(1-p) a^{2} ; \\
C_{1} & =\left[2+2 \rho_{12}+2 \rho_{13}\right] p(1-p) a^{2} ; \\
\lambda_{\text {eq, } 1} & =\frac{1}{3(1+2 \rho)},
\end{aligned}
$$

and

$\lambda_{\mathrm{dis}, 1}=\frac{2\left(1+\rho_{12}+\rho_{13}\right)}{6(1+2 \rho)}$.

If $\rho_{12}+\rho_{13}$ is replaced by $2 \rho$ (for illustrative purposes), then $\lambda_{\mathrm{dis}, 1}=0.33$, and each locus is assessed with an equal relative contribution to variance, whereas $\lambda_{\text {eq, } 1}$ understates the contribution of the locus to variability if disequilibrium is positive, but makes an overstatement if disequilibrium is negative."

5. A couple of lines after formula (12), replace: “...and off-diagonals $\rho_{j l} \sqrt{p_{j}\left(1-p_{j}\right) p_{l}\left(1-p_{l}\right)}$.” by “....and off-diagonals $2 \rho_{j l} \sqrt{p_{j}\left(1-p_{j}\right) p_{l}\left(1-p_{l}\right)}$."

6. In the "Results" section for a 3-locus model and positive linkage disequilibrium, replace:

$$
\begin{aligned}
C_{1}= & {\left[2 \times 0.5^{2}+0.8 \times 0.5 \sqrt{\left(0.25-\Delta^{2}\right)}\right.} \\
& \left.+0.6 \times 0.5 \sqrt{\left(0.25-4 \Delta^{2}\right)}\right] a^{2}, \\
C_{2}= & {\left[0.8 \times 0.5 \sqrt{\left(0.25-\Delta^{2}\right)}+2\left(0.25-\Delta^{2}\right)\right.} \\
& \left.+0.8 \sqrt{\left(0.25-\Delta^{2}\right)\left(0.25-4 \Delta^{2}\right)}\right] a^{2},
\end{aligned}
$$

and

$$
\begin{aligned}
C_{3}= & {\left[0.6 \times 0.5 \sqrt{(0.25-4 \Delta)^{2}}\right.} \\
& +0.8 \sqrt{\left(0.25-4 \Delta^{2}\right)\left(0.25-\Delta^{2}\right)} \\
& \left.+2\left(0.25-4 \Delta^{2}\right)\right] a^{2}
\end{aligned}
$$

by

$$
\begin{aligned}
C_{1}= & 2\left[0.5^{2}+0.8 \times 0.5 \sqrt{\left(0.25-\Delta^{2}\right)}\right. \\
& \left.+0.6 \times 0.5 \sqrt{\left(0.25-4 \Delta^{2}\right)}\right] a^{2},
\end{aligned}
$$

$$
\begin{aligned}
C_{2}= & 2\left[0.8 \times 0.5 \sqrt{\left(0.25-\Delta^{2}\right)}+\left(0.25-\Delta^{2}\right)\right. \\
& \left.+0.8 \sqrt{\left(0.25-\Delta^{2}\right)\left(0.25-4 \Delta^{2}\right)}\right] a^{2},
\end{aligned}
$$

and

$$
\begin{aligned}
C_{3}= & 2\left[0.6 \times 0.5 \sqrt{\left(0.25-4 \Delta^{2}\right)}\right. \\
& +0.8 \sqrt{\left(0.25-4 \Delta^{2}\right)\left(0.25-\Delta^{2}\right)} \\
& \left.+\left(0.25-4 \Delta^{2}\right)\right] a^{2},
\end{aligned}
$$

respectively.

7. Replace Fig. 3 by Fig. 3 in this Erratum.

8. Replace: "The relative contributions....at other values of $p$." by: "The relative contributions $\lambda_{\mathrm{eq}, j}$ and $\lambda_{\mathrm{dis}, j}$ of the three loci to variance were plotted against $\Delta$, as shown in Fig. 3 (left panel). The picture was clear: because LD was positive and strong, the standard formula based on $V_{j}$ produced a severe understatement of the contribution of any of the three loci to genetic variability at most frequencies. For example, in the case of locus 3 , its maximum contribution, as deemed by $V_{j}$, is attained when $\Delta=0(p=0.5)$, at nearly $13 \%$ of the variance (dotted green line). However, this locus makes a contribution of about $31-32 \%$ of the total genetic variance at frequencies near $p=0.50$ when indirect contributions stemming from $\mathrm{LD}$ (as conveyed by $C_{j}$ ) are taken into account. Importantly, note that while equilibrium formulae suggest that locus 1 is the most important contributor to variance at most allelic frequencies (dotted black line), this is not always so when both direct and indirect effects of a locus are brought into the picture. For example, the relative importance of loci 1 and 2 crisscross and locus 2 (solid red line) is the main contributor to variance at intermediate frequencies, but no so at other values of $p$."

9. In the "Results" section for a 3-locus model and negative linkage disequilibrium, replace:

$$
\begin{aligned}
C_{1}= & {\left[2 \times 0.5^{2}-0.7 \times 0.5 \sqrt{\left(0.25-\Delta^{2}\right)}\right.} \\
& \left.-0.3 \times 0.5 \sqrt{\left(0.25-4 \Delta^{2}\right)}\right] a^{2}, \\
C_{2}= & {\left[-0.7 \times 0.5 \sqrt{\left(0.25-\Delta^{2}\right)}+2\left(0.25-\Delta^{2}\right)\right.} \\
& \left.-0.2 \sqrt{\left(0.25-\Delta^{2}\right)\left(0.25-4 \Delta^{2}\right)}\right] a^{2},
\end{aligned}
$$



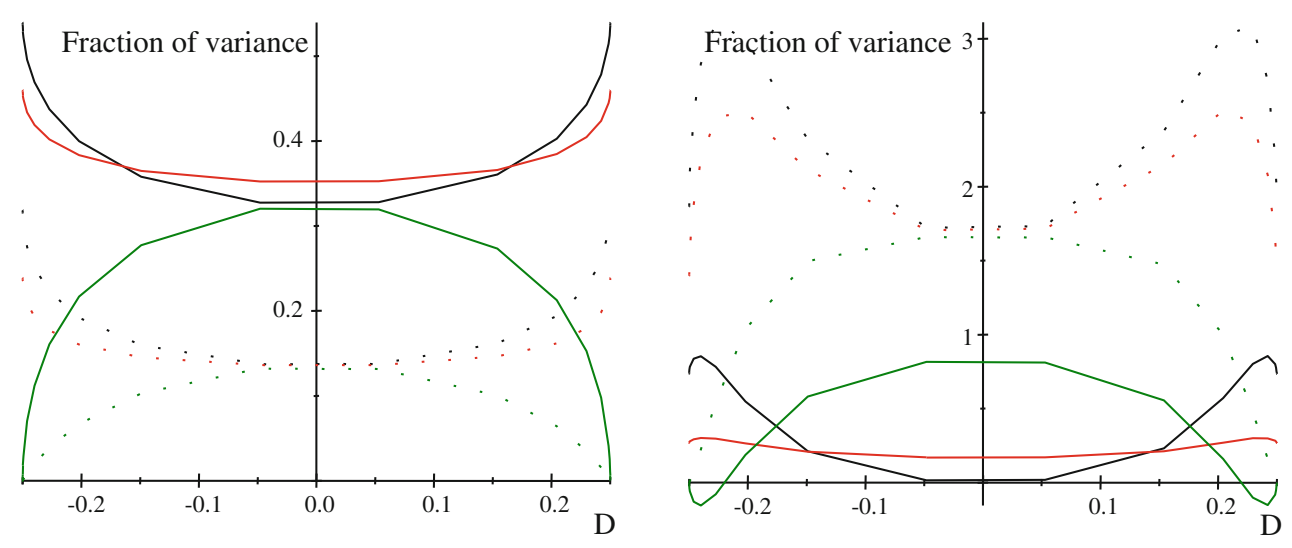

Fig. 3 Relative contribution to variance of three loci under positive (left panel) or negative (right panel) LD; dotted lines give the contributions as deemed by equilibrium formulae. Locus 1 black, Locus 2 red, Locus 3 green. $D$ departure of allelic frequency from 0.5 .

and

$$
\begin{aligned}
C_{3}= & {\left[-0.3 \times 0.5 \sqrt{(0.25-4 \Delta)^{2}}\right.} \\
& -0.2 \sqrt{\left(0.25-4 \Delta^{2}\right)\left(0.25-\Delta^{2}\right)} \\
& \left.+2\left(0.25-4 \Delta^{2}\right)\right] a^{2} .
\end{aligned}
$$

by

$$
\begin{aligned}
C_{1}= & 2\left[0.5^{2}-0.7 \times 0.5 \sqrt{\left(0.25-\Delta^{2}\right)}-0.3\right. \\
& \left.\times 0.5 \sqrt{\left(0.25-4 \Delta^{2}\right)}\right] a^{2}, \\
C_{2}= & 2\left[-0.7 \times 0.5 \sqrt{\left(0.25-\Delta^{2}\right)}+\left(0.25-\Delta^{2}\right)\right. \\
& \left.-0.2 \sqrt{\left(0.25-\Delta^{2}\right)\left(0.25-4 \Delta^{2}\right)}\right] a^{2},
\end{aligned}
$$

and

$$
\begin{aligned}
C_{3}= & 2\left[-0.3 \times 0.5 \sqrt{\left(0.25-4 \Delta^{2}\right)}\right. \\
& -0.2 \sqrt{\left(0.25-4 \Delta^{2}\right)\left(0.25-\Delta^{2}\right)} \\
& \left.+\left(0.25-4 \Delta^{2}\right)\right] a^{2},
\end{aligned}
$$

respectively.
10. Replace: "Figure 3 (right panel) depicts the relative importance of these three loci in terms of contribution to variance. The equilibrium formulae now overstate the relative importance of loci 1 and 3 , but slightly understate the contribution of locus 2 to variance. In this setting, negative disequilibrium results in negative contributions of locus 3 to variance at allelic frequencies that are approximately larger than 0.72 or smaller than about 0.28. The effect of negative disequilibrium on total variance also results in a reranking of loci." by:

"Figure 3 (right panel) depicts the relative importance of these three loci in terms of contribution to variance. The equilibrium formulae now overstate the relative importance of loci 1 and 2 at most frequencies, but understate the contribution of locus 3 to variance, especially at intermediate frequencies. In this setting, negative disequilibrium results in negative contributions of locus 3 to variance at allelic frequencies that are approximately larger than 0.72 or smaller than about 0.28 . The effect of negative disequilibrium on total variance also results in a reranking of loci at different allelic frequencies." 